\title{
Volume-ensemble search models for molecular replacement
}

\author{
Dmytro Guzenko ${ }^{1}$, Jose M. Duarte ${ }^{1}$, Stephen K. Burley ${ }^{1,2,3,4}$
}

\footnotetext{
${ }^{1}$ RCSB Protein Data Bank, San Diego Supercomputer Center, University of California, San Diego, La Jolla, CA 92093, USA

${ }^{2}$ RCSB Protein Data Bank, Institute for Quantitative Biomedicine, Rutgers, The State University of New Jersey, Piscataway, NJ 08854, USA

${ }^{3}$ Cancer Institute of New Jersey, Rutgers, The State University of New Jersey, New Brunswick, NJ 08903, USA

${ }^{4}$ Skaggs School of Pharmacy and Pharmaceutical Sciences, University of California, San Diego, La Jolla, CA 92093, USA
}

In molecular replacement structure determination, conformational flexibility of a target structure can be addressed using "ensemble" search models. Averaged electron density distributions obtained from superpositions of homologous structures typically yield more reliable scoring of rotation/translation search solutions versus results obtained with single-structure search models.

Computation of the ensemble model, however, often requires omission of flexible regions of structures, because only relatively rigid domains can be reliably aligned. Moreover, there is no unified method for incorporating structural information from other structural techniques that produce non-atomistic structure data, such as three-dimensional (3D) electron microscopy mass density maps or small-angle scattering envelopes.

We describe development of a novel, efficient rotation-invariant parameterization method for arbitrary volumetric models. Our procedure utilizes normalized 3D Zernike moments [1], yielding a set of independent parameters that allow faithful description of structure shapes at whatever level of precision is required. Potential scientific applications include any situation wherein systematic sampling of arbitrary 3D shapes is required (e.g., overcoming the phase problem in protein crystallography). Rotation-invariant parameters obtained from collections of homologous structures can be readily combined and interpolated. The resulting volume-ensemble molecular replacement model can also be parameterized to reflect regions of conformational flexibility and/or uncertainty within individual search model structures, where appropriate.

[1] Canterakis, N. (1999). 3D Zernike moments and Zernike affine invariants for 3D image analysis and recognition. In 11th Scandinavian Conference on Image Analysis, pp 85-93. 